\title{
Zytokin profiles in familial Mediterranean fever patients: IL-18 as a major cytokine and potential biomarker or target
}

\author{
A Igney-Oertel", J Henes, R Klein \\ From 8th International Congress of Familial Mediterranean Fever and Systemic Autoinflammatory Diseases \\ Dresden, Germany. 30 September - 3 October 2015
}

\section{Introduction}

Familial Mediterranean fever (FMF) is an autoinflammatory disease which is characterized by recurrent episodes of typical attacks with fever and abdominal pain. The pathophysiology shows an ineffective pyrin which leads to brakeless inflammation. It is not exactly known which factors lead to initiation of the attacks. Furthermore there seems to be sustained inflammation between the attacks.

IL-18 is acytokinethat belongs to theIL-1superfamily and is produced bymacrophagesand other cells. It plays an important role in the initiation and maturation of the inflammasome and is therefore able to induce severeinflammatory reactions.

\section{Objectives}

In our study we aimed to clarify the cytokine profiles of patients with genetically confirmed FMF in mainly attack free periods and compare it with healthy controls.

We evaluated type 1 cytokines: IFN-g, TNF-b, type 2 cytokines: IL-5, IL-10, IL-13 and other inflammatory cytokines: IL-1, IL-6, IL-17, IL-18, TNF-a and GMCSF.

\section{Patients and methods}

We examined 42 samples of 36 FMF patients between 07/ 2008 and $03 / 2015$. Six patients were evaluated at two time-points. Ten healthy subjects were examined as control group. The levels of the cytokines were determined by commercial ELISA kits.

\section{Results}

The mean age of the FMF patients was 34,1 years, $43 \%$ with homozygotic and $57 \%$ with combined heterozygotic mutations.

\footnotetext{
University Tuebingen, Medical Clinic, Hematology, Rheumatology, Tuebingen, Germany
}

Cite this article as: Igney-Oertel et al:: Zytokin profiles in familial

Mediterranean fever patients: IL-18 as a major cytokine and potential biomarker or target. Pediatric Rheumatology 2015 13(Suppl 1):P86.
Serum levels of IL-18 (normal range up to $400 \mathrm{pg} / \mathrm{ml}$ ) homozygotic patients were with $3654 \mathrm{pg} / \mathrm{ml}$ (mean value) higher than in patients with combined heterozygotic mutations $(1290 \mathrm{pg} / \mathrm{ml})$.

Patients with a complicated form of FMF (no colchiclear if this is a consequence of IL-1 antibody treatease-activity and heterozygotic mutations.

All other cytokines showed no significant differences which were evaluated at two time-points showed repeti-

\section{Conclusion}

ur results suggest a major role of Interleukin 18 in pathogenesis and disease course of familial MediterraPublished: 28 September 2015 